\title{
On the Complexity of Consistency and Complete State Coding for Signal Transition Graphs
}

\author{
Javier Esparza \\ Institute for Formal Methods in Computer Science \\ Univ. Stuttgart, Germany \\ esparza@informatik.uni-stuttgart.de
}

\author{
Petr Jančar* \\ Center of Applied Cybernetics \\ Dept of CS, TU Ostrava, Czechia \\ Petr.Jancar@vsb.cz
}

\author{
Alexander Miller \\ Institute for Formal Methods in Computer Science \\ Univ. Stuttgart, Germany \\ Alexander.Miller@informatik.uni-stuttgart.de
}

July 4, 2006

\begin{abstract}
Signal Transition Graphs (STGs) are a popular formalism for the specification of asynchronous circuits. A necessary condition for the implementability of an STG is the existence of a consistent and complete state encoding. For an important subclass of STGs, the marked graph STGs, we show that checking consistency is polynomial, but checking the existence of a complete state coding is co-NP-complete. In fact, co-NP-completeness already holds for acyclic and 1-bounded marked graph STGs and for live and 1-bounded marked graph STGs. We add some relevant results for free-choice, bounded, and general STGs.
\end{abstract}

\section{Introduction}

Signal transition graphs (STGs) are a popular formalism for specifying asynchronous circuits [3, 12]. They are Petri nets in which the firing of a transition is interpreted as rising or falling of a signal in the circuit. Not every STG can be implemented as a physical circuit. A central question related to implementability of an STG is whether it admits a so-called consistent and complete state coding. Most papers in the literature consider only the completeness part, assuming that the STG is already consistent, and call the existence of a complete state coding the CSC property. This property, and the stronger unique state coding property (USC property for short) have been studied in many papers (see e.g. $[1,9,10,11,14,16,17])$.

In this paper we reason about the computational complexity of deciding if a given STG has a consistent and complete state coding, viewing the consistency problem separately. We obtain new results for STGs whose underlying nets are marked graphs and free-choice nets; for completeness, we also sketch some straightforward results for STGs whose underlying nets are more general—bounded or even arbitrary.

\footnotetext{
*This author is supported by the Czech Ministry of Education, Grant No. 1M0567
} 
We first explore the consistency problem for marked graph STGs. In [7] a polynomial algorithm was given to check consistency of live, bounded, and cyclic free-choice STGs, which include live and bounded marked graph STGs as a subclass. Here we show that consistency is polynomial for arbitrary marked graph STGs by means of a new algorithm based on linear programming.

A natural question is whether these polynomiality results also hold for the CSC or USC problems (i.e., the problems of checking the CSC or USC properties), at least for the class of live and 1-bounded marked graph STGs. Our main result shows that both problems are co-NP-complete, and so that polynomial algorithms are unlikely. This result explains why the algorithms of $[1,9,10,11,14,16,17]$ have exponential runtime or can only decide some necessary or sufficient conditions for the CSC or USC properties to hold. These algorithms are discussed in detail in the final section.

Our co-NP-completeness result is rather robust. We prove that the CSC and USC problems remain co-NP-hard for 1-bounded and acyclic marked graph STGs, and that they remain in co-NP for arbitrary marked graph STGs and for live and bounded free-choice STGs.

Moving to more general classes, we show that the consistency, CSC and USC problems are PSPACE-complete for 1-bounded STGs, and that the consistency problem remains PSPACE-hard in the free-choice case. Finally, we clarify the relation between the consistency, USC and CSC problems for general STGs, and the fireability and reachability problems for general Petri nets.

The paper is structured as follows. Section 2 presents basic definitions and a characterization of consistency. Section 3 presents the results about marked-graph STGs; it is the core of the paper. Section 4 deals with free-choice and Section 5 with general STGs. Section 6 contains conclusions and discusses related work.

Remark. This report is the full version of the conference paper [8].

\section{Basic definitions}

A net is a triple $(P, T, F)$, where $P$ and $T$ are disjoint sets of places and transitions, respectively, and $F$ is a function $(P \times T) \cup(T \times P) \rightarrow\{0,1\}$. Places and transitions are generically called nodes; we also note that a net can be viewed as a (bipartite) graph. Places are graphically represented as circles; transitions are usually drawn like boxes, but we use just their labels in the figures. If $F(x, y)=1$ then we say that there is an $\operatorname{arc}$ from $x$ to $y$. The preset of a node $x$, denoted by ${ }^{\bullet} x$, is the set of its input nodes, i.e., the set $\{y \in P \cup T \mid F(y, x)=1\}$. The postset of $x$, denoted by $x^{\bullet}$, contains its output nodes, i.e., the set $\{y \in P \cup T \mid F(x, y)=1\}$.

A marking $M$ of a net $(P, T, F)$ is a mapping $P \rightarrow \mathbb{N}$ (where $\mathbb{N}$ denotes the set of natural numbers including 0). Graphically, a marking is represented by drawing $M(p)$ tokens on the circle representing the place $p$. A marking $M$ enables a transition $t$ if it puts at least one token on each place $p \in \bullet^{\bullet} t$, i.e., if $M(p) \geq 1$ for each $p \in \bullet^{\bullet} t$. If $t$ is enabled at $M$, then it can fire (or occur) and its firing (occurrence) leads to a new marking $L$, obtained by removing a token from each place in the preset of $t$, and adding a token to each place in its postset; formally, $L(p)=M(p)+F(t, p)-F(p, t)$ for every place $p . M \stackrel{t}{\longrightarrow}$ denotes that $t$ is enabled at $M$, and $M \stackrel{t}{\longrightarrow} M^{\prime}$ moreover denotes that firing $t$ leads to $M^{\prime}$.

The notation $M \stackrel{\sigma}{\longrightarrow}, M \stackrel{\sigma}{\longrightarrow} M^{\prime}$ is extended to finite sequences $\sigma \in T^{*}$ in the natural way. When $M \stackrel{\sigma}{\longrightarrow} M^{\prime}$, for $\sigma=t_{1} t_{2} \cdots t_{n}$, we sometimes speak about an occurrence sequence from $M$ to $M^{\prime}$, meaning the sequence

$$
M \stackrel{t_{1}}{\longrightarrow} M_{1} \stackrel{t_{2}}{\longrightarrow} \cdots M_{n-1} \stackrel{t_{n}}{\longrightarrow} M^{\prime}
$$


By the Parikh vector of $\sigma \in T^{*}$, denoted by $\vec{\sigma}$ or $P(\sigma)$, we mean the mapping $T \rightarrow \mathbb{N}$ such that $\vec{\sigma}(t)$ is the number of occurrences of $t$ in $\sigma$.

The incidence matrix of $N$ is the matrix $C_{N}: P \times T \rightarrow\{-1,0,+1\}$ given by $C_{N}(p, t)=F(t, p)-$ $F(p, t)$. We note that if $M \stackrel{\sigma}{\longrightarrow} M^{\prime}$ then $M+C_{N} \cdot \vec{\sigma}=M^{\prime}$. (We naturally identify the mapping $\vec{\sigma}$ with a (nonnegative integer) vector; that's why we use the term 'Parikh vector'.)

A Petri net is a pair $\left(N, M_{0}\right)$ where $N$ is a net and $M_{0}$ is a marking of $N$, called the initial marking. A marking $M$ is called reachable if there exists an occurrence sequence from $M_{0}$ to $M$; we also denote this by $M_{0} \rightarrow^{*} M$. We call

$$
M_{0}+C_{N} \cdot X \geq 0
$$

the marking inequation. We note that $M_{0} \stackrel{\sigma}{\longrightarrow} M$ implies $M_{0}+C_{N} \cdot \vec{\sigma}=M$; $\vec{\sigma}$ is thus a (nonnegative integer) solution of the marking inequation.

A marking $M$ of a net $N$ is $n$-bounded if $M(p) \leq n$ for every place $p$. A Petri net $\left(N, M_{0}\right)$ is $n$-bounded if all its reachable markings are $n$-bounded.

A transition $t$ is fireable in $\left(N, M_{0}\right)$ if there is $\sigma$ such that $M_{0} \stackrel{\sigma}{\longrightarrow} M$ and $M \stackrel{t}{\longrightarrow}$. A Petri net $\left(N, M_{0}\right)$ is live if each transition $t$ is fireable in $(N, M)$ for each $M$ reachable from $M_{0}$. A transition is dead at a marking $M$ if $t$ is not fireable in $(N, M)$.

A net $N$ is called a marked graph if every place has at most one input and at most one output transition. $N=(P, T, F)$ is a free-choice net if: for each place $p$ and every transition $t$, if $F(p, t)=1$ then $F\left(p^{\prime}, t^{\prime}\right)=1$ for every $p^{\prime} \in{ }^{\bullet} t, t^{\prime} \in p^{\bullet}$. In a free-choice net, if some output transition of a place is enabled at a marking, then all its output transitions are enabled, and it is possible to "freely" choose among them.

Signal transition graphs. Let $A=\left\{a_{1}, \ldots, a_{n}\right\}$ be a set (alphabet) of signals partitioned into input and output signals. Rising and falling of a signal $a$ is denoted by $a^{+}$and $a^{-}$, respectively. (In some proofs we also use the notation $+a$ and $-a$, which is more convenient for using sub- and superscripts.) We call an element of $\mathcal{L}=A \times\{+,-\}$ a label. A signal transition graph (STG) is a triple $S=\left(N, M_{0}, \ell\right)$, where $\left(N, M_{0}\right)$ is a Petri net and $\ell$ is a labelling function that assigns to each transition of $N$ a label in $\mathcal{L}$.

A signal transition graph is a specification of the behaviour of the circuit under some assumptions on the environment. An STG $S$ is implementable if there exists a state coding mapping $\lambda$ (we also use the term binary encoding) that associates to each reachable marking $M$ a vector of signal values $\lambda(M) \in\{0,1\}^{n}$ satisfying the following two properties:

(1) Consistency. If $M \stackrel{t}{\longrightarrow} L$ and $t$ is labelled by $a_{i}^{+}$, then the $i$-th components of $\lambda(M)$ and $\lambda(L)$ are 0 and 1 , respectively, and all other components have the same value in $\lambda(M)$ and $\lambda(L)$. If $t$ is labelled by $a_{i}^{-}$, then the $i$-th components of $\lambda(M)$ and $\lambda(L)$ are 1 and 0 , respectively, and all other components have the same value in $\lambda(M)$ and $\lambda(L)$.

(2) Completeness: if two different reachable markings $M, L$ satisfy $\lambda(M)=\lambda(L)$, then they enable exactly the same output labels.

Consistency is obviously necessary for implementability. Completeness is necessary because the state of an implementation is completely determined by the signal values of all signals. Therefore, if some output signal is enabled at $M$ but not at $L, M$ and $L$ must correspond to different states of the implementation, and so they must differ in the value of at least one signal. 
We define the consistency problem as the problem of deciding if a given STG is consistent, i.e., if it admits a binary encoding $\lambda$ satisfying (1). The Complete State Coding problem, CSC problem for short, is the problem to decide if a given STG (usually already assumed consistent) has the CSC property, i.e., admits a binary encoding satisfying (1) and (2). A stricter version is the USC problem (unique state coding) where we ask if a given STG has the USC property, i.e., admits an injective binary encoding $\lambda$ satisfying (1) (thus $\lambda(M) \neq \lambda(L)$ for any two different reachable $M, L$ ).

STGs naturally inherit many notions from their underlying (Petri) nets. We already used this when speaking about 'enabling a label', e.g. $M \stackrel{a^{+}}{\longrightarrow}$ (meaning that $M$ enables a transition with label $a^{+}$). Thus we will freely speak about $n$-bounded, live, marked graph, or free-choice STGs, etc. We can also use notions like $a$ is dead at $M$ (meaning that each transition with label $a^{+}$or $a^{-}$is dead at $M$ ).

We also freely use notation like $M \stackrel{u}{\longrightarrow} M^{\prime}$ for sequences of labels (meaning that there is a transition sequence $\sigma=t_{1} t_{2} \cdots t_{m}$ such that $M \stackrel{\sigma}{\longrightarrow} M^{\prime}$ and $\left.u=\ell\left(t_{1}\right) \ell\left(t_{2}\right) \cdots \ell\left(t_{m}\right)\right)$. We can occasionally even mix, and consider $u$ as a sequence of transitions and labels, when this should not cause confusion. We also use expressions like $u$ is $a$-free, meaning that there is no $a^{+}$nor $a^{-}$in $u$; and if $u$ contains transitions, we mean that those transitions do not have labels $a^{+}, a^{-}$. Recall that $P(u)$ denotes the Parikh vector of $u$; We denote by $P(u)\left(a^{+}\right)$the number of transitions with label $a^{+}$in $u$.

Finally we note that since the circuit implementation of an STG can be seen as a finite object with at most $2^{n}$ states, where $n$ is the number of signals, STGs used in practice are bounded, most of them are even 1-bounded; but in principle unbounded STGs can make sense.

We finish the section by a characterization of consistency, i.e., we look in more detail on when an STG is inconsistent.

Proposition 2.1 An STG $S=\left(N, M_{0}, \ell\right)$ is inconsistent (i.e., it admits no consistent binary encoding) iff there is

$$
\begin{gathered}
\text { a pair }(M, a) \\
\text { where } M_{0} \rightarrow^{*} M \text { and a is a signal }
\end{gathered}
$$

such that one of the following conditions holds:

(1) $M$ enables $u a^{+}$and $v a^{-}$

for some a-free sequences $u, v$,

(2) $M$ enables $a^{+} u a^{+}$or $a^{-} u a^{-}$

for some a-free sequence $u$,

(3) $M$ is reachable by $w_{1} a^{+} u$ and by $w_{2} a^{-} v$

for some a-free sequences $u, v$ (and some $w_{1}, w_{2}$ ).

Proof: If there is a pair $(M, a)$ such that one of the conditions holds then $S$ is obviously inconsistent.

If there is no such pair then we can (soundly) define the following (partial) encoding $\lambda$ :

For each reachable $M$ and signal $a$ we put

- $\lambda(M)(a)=0$ if $M$ enables $u a^{+}$for an $a$-free sequence $u$

- $\lambda(M)(a)=1$ if $M$ enables $u a^{-}$for an $a$-free sequence $u$

We note that if $\lambda(M)(a)$ is (sofar) undefined then $M$ is $a$-dead; we then put 
- $\lambda(M)(a)=1$ when $M$ can be reached by $w a^{+} u$ for some $a$-free sequence $u$

- $\lambda(M)(a)=0$ when $M$ can be reached by $w a^{-} u$ for some $a$-free sequence $u$

- $\lambda(M)(a)=0$ otherwise (i.e., when $M$ is reachable only by $a$-free sequences)

(In the last case we could use $\lambda(M)(a)=1$ as well.)

One can easily check that $\lambda$ is a consistent binary encoding.

\section{Marked graphs}

In this section we show that consistency can be decided in polynomial time for all marked graph STGs and that both the CSC problem and the USC problem are co-NP-complete for them, even in the case of 1-bounded acyclic marked graphs and in the case of live 1-bounded marked graphs.

\subsection{Consistency}

In [7] it is shown that consistency of live, bounded, and cyclic free-choice STGs can be decided in polynomial time. (A Petri net is cyclic if the initial marking is reachable from every reachable marking, i.e., if it is always possible to return to the initial marking). Since live and bounded marked graphs are always cyclic (see for instance [4]), and marked graphs are a special case of free-choice nets, [7] provides a polynomial algorithm deciding consistency of live and bounded marked graph STGs. We now show a polynomial algorithm for all marked graph STGs.

We start by recalling some simple properties of marked graphs and derive a simpler variant of Proposition 2.1, valid for marked graphs. One such property is that if $M$ enables a sequence with $n$ occurrences of $t$ and $M \stackrel{t^{\prime}}{\longrightarrow} M^{\prime}$ for $t^{\prime} \neq t$ then $M^{\prime}$ enables a sequence with $n$ occurrences of $t$ as well; if $t^{\prime}=t$ then $M^{\prime}$ enables a sequence with $n-1$ occurrences of $t$.

By $P(u)(t)$ we denote the number of occurrences of $t$ in a transition sequence $u$ ( $P$ stands for the Parikh vector).

Claim 3.1 Let $M$ be a marking of a marked graph. If $M \stackrel{u}{\longrightarrow} M_{1}$ and $M \stackrel{v}{\longrightarrow} M_{2}$ then $M \stackrel{w}{\longrightarrow} M^{\prime}$ for some $w$ and $M^{\prime}$ such that

$$
\forall t: P(w)(t)=\max \{P(u)(t), P(v)(t)\} .
$$

Moreover, if $M_{1} \stackrel{t}{\rightarrow}$ and $P(v)(t) \leq P(u)(t)$ then $M^{\prime} \stackrel{t}{\rightarrow}$.

Proof: We can perform $u$ and then a sequence consisting of $P(v)(t)-P(u)(t)$ occurrences of each $t$ for which $P(u)(t)<P(v)(t)$.

Slightly abusing notation, by $\max (u, v)$ we will denote the $w$ guaranteed by the claim.

Proposition 3.1 A marked graph $S T G S=\left(N, M_{0}, \ell\right)$ is inconsistent iff one of the following conditions holds: 
(1') there is a reachable $M\left(M_{0} \rightarrow^{*} M\right)$ such that

$M \stackrel{a^{+}}{\longrightarrow}$ and $M \stackrel{a^{-}}{\longrightarrow}$ for some signal $a$,

(2') there is a reachable $M$ such that

$M \stackrel{a^{+} u a^{+}}{\longrightarrow}$ or $M \stackrel{a^{-} u a^{-}}{\longrightarrow}$

for some signal $a$ and some a-free sequence $u$.

Proof: If (1') or (2') holds then $S$ is obviously inconsistent.

Now assume that $S$ is inconsistent. Then we know that there is a reachable $M$ and a signal $a$ such that one of the conditions (1), (2), (3) of Proposition 2.1 holds. It is sufficient to show that this implies (1') or (2').

If $(M, a)$ satisfies (2) then (2') holds. If $(M, a)$ satisfies (1), i.e. $M \stackrel{u a^{+}}{\longrightarrow}$ and $M \stackrel{v a^{-}}{\longrightarrow}$ for $a$-free sequences $u, v$, then $M \stackrel{\max (u, v)}{\longrightarrow} M^{\prime}$ and $M^{\prime} \stackrel{a^{+}}{\longrightarrow}, M^{\prime} \stackrel{a^{-}}{\longrightarrow}$ (recall Claim 3.1); thus (1') holds.

We finish by deriving a contradiction from the assumption that the inconsistency of $S$ can not be shown by using (1) nor (2) while we have $(M, a)$ satisfying (3). Hence

$$
M_{0} \stackrel{w_{1} t_{1} u}{\longrightarrow} M \text { and } M_{0} \stackrel{w_{2} t_{2} v}{\longrightarrow} M
$$

where $\ell\left(t_{1}\right)=a^{+}, \ell\left(t_{2}\right)=a^{-}$, and $u, v$ are $a$-free.

Necessarily, all transitions labelled by $a^{+}$or $a^{-}$are dead in $M-$ otherwise there would exist $\left(M^{\prime}, a\right)$ satisfying (2). Thus both $t_{1}, t_{2}$ are dead in $M$, which means that $w_{1}$ contains the maximal possible number of occurrences of $t_{2}$, while $w_{2}$ contains the maximal possible number of occurrences of $t_{1}$.

Let $w_{1}=u_{1} t_{2} v_{1}$ where $v_{1}$ is $t_{2}$-free. Similarly $w_{2}=u_{2} t_{1} v_{2}$ where $v_{2}$ is $t_{1}$-free. We note that $P\left(u_{2}\right)\left(t_{2}\right) \leq P\left(u_{1}\right)\left(t_{2}\right)$, and $P\left(u_{1}\right)\left(t_{1}\right) \leq P\left(u_{2}\right)\left(t_{1}\right)$.

Hence $M_{0} \stackrel{\max \left(u_{1}, u_{2}\right)}{\longrightarrow} M^{\prime}$ where $M^{\prime}$ enables both $t_{1}$ and $t_{2}$, so we have both $M^{\prime} \stackrel{a^{+}}{\longrightarrow}$ and $M^{\prime} \stackrel{a^{-}}{\longrightarrow}$. Thus $\left(M^{\prime}, a\right)$ satisfies $(1)-$ a contradiction.

It is now sufficient to show that conditions (1'), (2') of Proposition 3.1 can be checked in polynomial time.

To this aim, we recall further useful observations about marked graphs. We note that, given a marked graph STG $S=\left(N, M_{0}, \ell\right)$, we can check in polynomial time if there is a circuit of $N$ which is not marked at $M_{0}$ (i.e., its places have no tokens in $M_{0}$ ). The places of such a circuit can be safely removed, since no transition in the circuit can ever occur.

We call a marked graph $\left(N, M_{0}\right)$ normalized if every circuit of $N$ is marked at $M_{0}$.

Claim 3.2 Let $\left(N, M_{0}\right)$ be a normalized marked graph, and consider the inequation $M_{0}+C_{N} \cdot X \geq 0$, where $C_{N}$ is the incidence matrix of $N$. An integer vector $X_{0} \geq 0$ is a solution of this inequation if and only if $M_{0} \stackrel{\sigma}{\longrightarrow}$ for a transition sequence $\sigma$ whose Parikh vector is $X_{0}$.

Moreover, if $M_{0} \stackrel{\sigma}{\longrightarrow} M$ then $M_{0}+C_{N} \cdot X_{0}=M$.

Proof: The only nonobvious claim is that a solution $X_{0}$ implies the existence of an appropriate $\sigma$. But this can be done easily by induction on $\left|X_{0}\right|$ : among the transitions $t$ with $X_{0}(t) \geq 1$, some 
must be enabled at $M_{0}$ - otherwise we would construct a circuit unmarked in $M_{0}$; we can fire such a transition and use the induction hypothesis.

Now we come to the polynomiality claims, which can be quickly established by using linear programming (which is a well-known polynomial problem).

Proposition 3.2 For normalized marked graph STGs, checking (1') of Proposition 3.1 can be done in polynomial time.

Proof: Let $S=\left(N, M_{0}, \ell\right)$ be a normalized marked graph STG. If $S$ satisfies (1') then $N$ contains transitions $t_{1}, t_{2}$ with $\ell\left(t_{1}\right)=a^{+}$and $\ell\left(t_{2}\right)=a^{-}$such that there is $\sigma$ for which

$$
M_{0} \stackrel{\sigma}{\longrightarrow} M, M \stackrel{t_{1}}{\longrightarrow}, M \stackrel{t_{2}}{\longrightarrow} .
$$

We note that $M \geq M_{t_{1}}+M_{t_{2}}$ where $M_{t}$ denotes the marking that puts one token in each input place of $t$ and no tokens elsewhere. The Parikh vector of $\sigma$ is thus a solution of the linear inequation

$$
M_{0}+C_{N} \cdot X \geq M_{t_{1}}+M_{t_{2}}
$$

On the other hand, if the inequation has a nonnegative, rational solution $X_{0}$ then the integer vector $\left\lfloor X_{0}\right\rfloor$ is also a solution, as one can easily check. Claim 3.2 then guarantees the existence of an appropriate $\sigma$, meaning that $S$ satisfies (1').

Thus checking (1') can be done by solving the inequations for all appropriate pairs $t_{1}, t_{2}$.

Proposition 3.3 For normalized marked graph STGs not satisfying (1'), checking (2') can be done in polynomial time.

Proof: Let $S=\left(N, M_{0}, \ell\right)$ be a normalized marked graph STG which does not satisfy (1'); i.e., no reachable $M$ can enable both $a^{+}$and $a^{-}$. From this we can derive that $\left(M_{0}, a\right)$ does not satisfy (1) of Proposition 2.1. Therefore, in every occurrence sequence containing occurrences of the signal $a$, the first occurrence of $a$ always has the same sign. Which sign this is, + or - , can be determined very efficiently, e.g. by firing any maximal transition sequence in which each transition of $S$ occurs at most once (such a sequence contains all transitions that can ever be enabled).

Consider signal $a$, and assume we have found that $a^{+}$is fireable as the first of $a^{+}, a^{-}$. (The case with $a^{-}$being the first is similar.)

Let us now solve the linear programming problems

$$
\begin{array}{ll}
\text { maximize } & f(X) \\
\text { subject to } & X \geq 0, M_{0}+C_{N} \cdot X \geq 0 \\
\text { minimize } & f(Y) \\
\text { subject to } & Y \geq 0, M_{0}+C_{N} \cdot Y \geq 0
\end{array}
$$

where

$$
f(X)=\sum_{t \in \ell^{-1}\left(a^{+}\right)} X(t)-\sum_{t \in \ell^{-1}\left(a^{-}\right)} X(t)
$$


If we find that it is NOT the case that both problems have optimal solutions $X_{o p}, Y_{o p}$ with $f\left(X_{o p}\right)=1$ and $f\left(Y_{o p}\right)=0$ then we claim ' $(2$ ') holds'.

To check (2'), we run the above procedure for each signal $a$ separately, and claim that (2') holds when one signal gives rise to this claim, otherwise we claim that (2') does not hold. The overall time of this algorithm is surely polynomial; it remains to show its correctness.

Let us again consider signal $a$ where $a^{+}$is fireable as the first (of $a^{+}, a^{-}$). It is obvious that condition (2') holds for signal $a$ iff there is a transition sequence $\sigma$, with Parikh vector $X$, such that $M_{0} \stackrel{\sigma}{\longrightarrow}$ and $f(X) \geq 2$ (there are two $a^{+}$'s without any $a^{-}$in-between) or $f(X) \leq-1$ (two $a^{-}$'s without any $a^{+}$in-between).

So if (2') holds for signal $a$ then the procedure for $a$ surely gives rise to the claim '(2') holds'.

If (2') does not hold for $a$ then we have $f(X) \in\{0,1\}$ for each integer admissible solution $X$ (due to Claim 3.2). We want to show that the procedure for $a$ finds some optimal solutions $X_{o p}, Y_{o p}$ with $f\left(X_{o p}\right)=1$ and $f\left(Y_{o p}\right)=0$ (and thus does not give rise to the claim '(2') holds').

To see this we recall that all solutions of $X \geq 0, M_{0}+C_{N} \cdot X \geq 0$ constitute a polyhedron. The optimal solutions $X_{o p}, Y_{o p}$ exist iff $f(X)$ is bounded from above and from below on the polyhedron, and then such solutions can be found in the extremal points. The fact that $f(X) \in\{0,1\}$ for all integer $X$ easily implies that $f(X)$ is bounded for all (admissible) $X$; thus the optimal solutions exist.

We now note that every row of $C_{N}$ contains at most one +1 and at most one -1 , which means that matrix $C_{N}$ is (totally) unimodular. Hence the extremal points of the polyhedron are integer vectors (cf., e.g., [13]). Thus the procedure for $a$ indeed finds some optimal solutions $X_{o p}, Y_{o p}$ with $f\left(X_{o p}\right)=1$ and $f\left(Y_{o p}\right)=0$.

Theorem 3.1 Consistency of marked graph STGs can be decided in polynomial time.

Proof: The polynomial algorithm first normalizes the STG and then uses the algorithms guaranteed by Propositions 3.2 and 3.3 to check if one of the conditions (1'), (2') of Proposition 3.1 holds.

\subsection{Complete state coding}

In this subsection we show the announced co-NP-completeness results for the CSC problem and the USC problem on (consistent) marked graph STGs.

The next lemma is the main technical result of the paper. We say that an occurrence sequence is balanced if for every signal $a$ the sequence contains the same number of occurrences of transitions labelled by $a^{+}$and of transitions labelled by $a^{-}$.

Lemma 3.1 The following problem is NP-complete:

Instance: a (consistent) $S T G S=\left(N, M_{0}, \ell\right)$ such that $\left(N, M_{0}\right)$ is a 1-bounded, acyclic marked graph.

Question: is there an occurrence sequence $M_{0} \stackrel{\sigma}{\longrightarrow} M_{1} \stackrel{\tau}{\longrightarrow} M_{2}$ of $S$ such that $\tau$ is nonempty and balanced?

Proof: Membership in NP is clear: In any net $\left(N, M_{0}\right)$ which is 1-bounded and acyclic, each transition can appear at most once in any occurrence sequence. So a nondeterministic algorithm can just 
guess a sequence $\sigma \tau$ of pairwise distinct transitions and verify that it is performable from $M_{0}$ and that $\tau$ is nonempty and balanced.

The main point is NP-hardness, which we show by a reduction from CNF-SAT. Let $\varphi$ be a boolean formula in conjunctive normal form

- with $m$ clauses $c_{1}, \ldots, c_{m}$,

- and $n$ variables $x_{1}, \ldots, x_{n}$.

(E.g., formula $\left(x_{1} \vee \overline{x_{2}} \vee x_{3}\right) \wedge\left(x_{2} \vee \overline{x_{4}}\right)$ has 2 clauses and 4 variables.)

Our aim is to show a polynomial construction of a certain STG $S_{\varphi}=\left(N, M_{0}, \ell\right)$, with $\left(N, M_{0}\right)$ being a 1-bounded acyclic marked graph, so that $\varphi$ is satisfiable iff $S_{\varphi}$ admits $M_{0} \stackrel{\sigma}{\longrightarrow} M_{1} \stackrel{\tau}{\longrightarrow} M_{2}$ for some sequence $\sigma$ and some nonempty balanced sequence $\tau$.

The construction is based on the fact that there is a truth assignment

$$
\mathcal{A}:\left\{x_{1}, x_{2}, \ldots, x_{n}\right\} \rightarrow\{0,1\}
$$

satisfying $\varphi$ if and only if there is a consistent choice of literals, by which we mean a mapping

$$
l:\left\{c_{1}, c_{2}, \ldots, c_{m}\right\} \rightarrow\left\{x_{1}, \overline{x_{1}}, x_{2}, \overline{x_{2}}, \ldots, x_{n}, \overline{x_{n}}\right\}
$$

attaching to each clause $c_{i}$ one of its literals, denoted $l\left(c_{i}\right)$, in such a way that $l\left(c_{i}\right) \neq \overline{l\left(c_{j}\right)}$ for all $i, j$ (i.e., it is forbidden that one clause 'chooses' $x$ while another clause 'chooses' $\bar{x}$ ).

We can easily observe that any consistent choice of literals $l$ naturally provides a satisfying truth assignment $\mathcal{A}$ (which can be specified arbitrarily for variables not appearing in the range of $l$ ); and any satisfying truth assignment enables to define (maybe several) consistent choices of literals.

We now describe the STG $S_{\varphi}$, providing also informal comments which will ease the later correctness proof. Figure 1 shows the overall structure of $S_{\varphi}$.

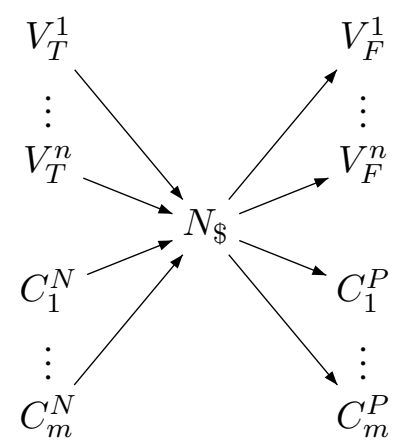

Figure 1: The overall structure of $S_{\varphi}$

We need a few remarks about the notation. We construct $S_{\varphi}=\left(N, M_{0}, \ell\right)$ where $N$ is an acyclic marked graph. All the minimal elements with respect to the flow relation will be places, and precisely those places will be initially marked (i.e., each will carry one token). We say that there is an arc from transition $t_{1}$ to transition $t_{2}$ when there is an (intermediate) place $p$ (initially unmarked) and arcs $t_{1} \rightarrow p, p \rightarrow t_{2}$. (This is, in fact, a usual convention which we also use for drawing marked graphs.)

Each symbol of Figure 1 (i.e., each $V_{T}^{1}, \ldots, C_{m}^{P}$ ) stands for an acyclic marked graph. The arrow $V_{T}^{1} \rightarrow N_{\$}$ has the following meaning: $V_{T}^{1}$ has a transition $t$ which is the unique maximal element in 
$V_{T}^{1}$ (w.r.t. the order induced by the flow relation), $N_{\$}$ has a transition $u$ which is the unique minimal element in $N_{\$}$, and the (overall) net $N$ contains an arc leading from $t$ to $u$ (with an intermediate place-using our convention). The meaning of the other arrows in the structure is analogous.

It will be clear (after we finish the construction) that any complete behaviour of $S_{\varphi}$ can be divided into three phases:

I. first, all transitions in $V_{T}^{1}, \ldots, V_{T}^{n}, C_{1}^{N}, \ldots, C_{m}^{N}$ occur,

II. then all transitions of $N_{\$}$ follow,

III. and finally all transitions in $V_{F}^{1}, \ldots, V_{F}^{n}, C_{1}^{P}, \ldots, C_{m}^{P}$ occur.

The complete behaviours of $S_{\varphi}$ differ only in the order in which transitions occur in the phases I and III. We proceed to describe the marked graphs corresponding to $N_{\$}, V_{T}^{1}, \ldots, V_{T}^{n}, C_{1}^{N}, \ldots, C_{m}^{N}$. Since we need to use both sub- and superscripts, we change the notation and write $+a$ and $-a$ instead of $a^{+}$ and $a^{-}$. The net $N_{\$}$, enabled after the whole phase I is finished, has one single (complete) behaviour, shown in Figure 2.

$$
+\$-x^{1}-x^{2} \cdots-x^{n}-c_{1}-c_{2} \cdots-c_{m}-\$
$$

Figure 2: (Linear) behaviour of $N_{\$}$

This means that the signal set of $S_{\varphi}$ contains (among others):

- a signal $c_{i}$ for every clause $(1 \leq i \leq m)$;

- a signal $x^{j}$ for every variable $(1 \leq j \leq n)$;

- a (special) signal $\$$.

Signal $\$$ will not appear anywhere else but in $N_{\$}$. It will be the case that any nonempty balanced sequence must include all transitions of $N_{\$}$, and so such a sequence will necessarily contain the whole phase II.

For the rest of the proof let bal denote any non-empty and balanced sequence such that $M_{0} \stackrel{\sigma}{\longrightarrow} M_{1} \stackrel{b a l}{\longrightarrow} M_{2}$. In bal, each falling $-x^{j}(1 \leq j \leq n)$ must be compensated by a raising $+x^{j}$; the label $+x^{j}$ will appear just on the maximal (i.e., the last) transition of $V_{T}^{j}$ (cf. Figure 3 ) and on the minimal (i.e., the first) transition of $V_{F}^{j}$ (cf. Figure 4). So precisely one of the subnets $V_{T}^{j}, V_{F}^{j}$ will contribute to bal. We interpret this as 'choosing' a truth assignment $\mathcal{A}$.

Similarly, each falling $-c_{i}(1 \leq i \leq m)$ must be compensated by a raising $+c_{i}$; the label $+c_{i}$ will appear just once in $C_{i}^{N}$ and once in $C_{i}^{P}$, now 'almost' as the last transition and 'almost' as the first transition, respectively. Again, exactly one of the subnets $C_{i}^{N}, C_{i}^{P}$ will contribute to bal.

Now we continue with the details of our construction. We extend the signal set used so far by

- a signal $p_{i}^{j}$ for each pair $i, j(1 \leq i \leq m, 1 \leq j \leq n)$ such that clause $c_{i}$ contains literal $x_{j}(p$ stands for 'positive');

- a signal $n_{i}^{j}$ for each pair $i, j(1 \leq i \leq m, 1 \leq j \leq n)$ such that clause $c_{i}$ contains literal $\overline{x_{j}}(n$ stands for 'negative'). 
(As usual, we can assume that no clause $c_{i}$ of formula $\varphi$ contains a complementary pair of literals.)

Given $j(1 \leq j \leq n)$, let $\left\{c_{i_{1}}, c_{i_{2}}, \ldots, c_{i_{a}}\right\}$ be the set of clauses containing (positive) literal $x_{j}$. The (sub)net $V_{T}^{j}$ (representing setting $x_{j}$ to 'true') is depicted in Figure 3. Thus $V_{T}^{j}$ 'emits' labels

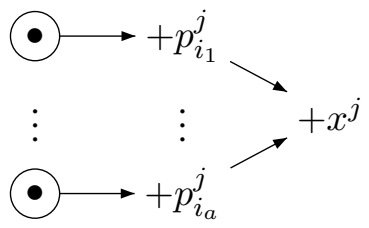

Figure 3: The net $V_{T}^{j}$

$+p_{i_{1}}^{j},+p_{i_{2}}^{j}, \ldots,+p_{i_{a}}^{j}$ in any order, and then finishes by $+x^{j}$.

Now let $\left\{c_{k_{1}}, c_{k_{2}}, \ldots, c_{k_{b}}\right\}$ be the set of clauses containing (negative) literal $\overline{x_{j}}$. The (sub)net $V_{F}^{j}$ (representing setting $x_{j}$ to 'false') is depicted in Figure 4. Thus, after the label $-\$$ of $N_{\$}$ occurs, $V_{F}^{j}$

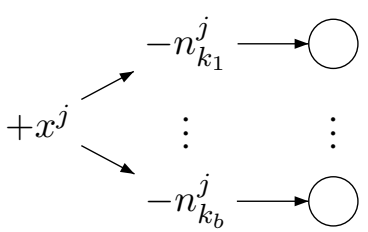

Figure 4: The net $V_{F}^{j}$

'emits' label $+x^{j}$ and then labels $-n_{k_{1}}^{j},-n_{k_{2}}^{j}, \ldots,-n_{k_{b}}^{j}$ in any order.

We now define the subnets $C_{i}^{N}, C_{i}^{P}$. Recall that the sequence $b a l$ will contain either transitions of $C_{i}^{N}$ or $C_{i}^{P}$, but not of both. This corresponds to 'choosing' either a positive or a negative literal $l\left(c_{i}\right)$ from $c_{i}$. Which literal is chosen will depend on which transitions of the corresponding net occur in $b a l$, and is explained later.

The nets $C_{i}^{N}$ and $C_{i}^{P}$ have no concurrency. They use additional 'parenthetical' signals. More precisely, we enhance the signal set by

- a signal $\square_{i}^{j}$ for each pair $i, j, 1 \leq i \leq m$ and $1 \leq j \leq n_{i}$, where $n_{i}$ is the number of negative literals in $c_{i}$;

- a signal $\triangle_{i}^{j}$ for each pair $i, j, 1 \leq i \leq m$ and $1 \leq j \leq p_{i}$, where $p_{i}$ is the number of positive literals in $c_{i}$.

Given $i(1 \leq i \leq m)$, let $\left\{\overline{x_{j_{1}}}, \overline{x_{j_{2}}}, \ldots, \overline{x_{j_{a}}}\right\}$ be the set of negative literals of the clause $c_{i}$.

The (sub)net $C_{i}^{N}$ has a (marked) place as the least element (w.r.t. the flow relation). And the only (complete) behaviour of $C_{i}^{N}$ is the sequence of labels shown in Figure 5. The key observation is that if the label $+c_{i}$ of $C_{i}^{N}$ belongs to the balanced sequence $b a l$, then bal must also contain $-\square_{i}^{a}$, and thus, by balancedness, also $+\square_{i}^{a}$. But then $b a l$ also contains $+n_{i}^{j_{a}}$, and so it must also contain $-n_{i}^{j_{a}}$. If we add the label $-n_{i}^{j_{a}}$ of $C_{i}^{N}$ to $b a l$, then we are forced to add $-\square_{i}^{a-1}$ as well, and thus also $+\square_{i}^{a-1}$ and $+n_{i}^{j_{a-1}}$; etc. So if labels of $C_{i}^{N}$ occur in bal, then bal contains an occurrence of some $+n_{i}^{j}$, where $\overline{x_{j}}$ 


$$
\begin{gathered}
+\square_{i}^{1}+n_{i}^{j_{1}}-n_{i}^{j_{2}}-\square_{i}^{1}+\square_{i}^{2}+n_{i}^{j_{2}}-n_{i}^{j_{3}}-\square_{i}^{2} \cdots \\
\cdots+\square_{i}^{a-1}+n_{i}^{j_{a-1}}-n_{i}^{j_{a}}-\square_{i}^{a-1}+\square_{i}^{a}+n_{i}^{j_{a}}+c_{i}-\square_{i}^{a}
\end{gathered}
$$

Figure 5: (Linear) behaviour of $C_{i}^{N}$

is a literal of $c_{i}$, such that the 'balancing' occurrence of $-n_{i}^{j}$ does not come from $C_{i}^{N}$, and so it must come from $V_{F}^{j}$. We interpret this as 'choosing' the literal $\overline{x_{j}}$ of $c_{i}$, i.e., as setting $l\left(c_{i}\right)=\overline{x_{j}}$.

The (sub)net $C_{i}^{P}$ is similar. We let $\left\{x_{k_{1}}, x_{k_{2}}, \ldots, x_{k_{b}}\right\}$ be the set of positive literals of the clause $c_{i}$. The least element of $C_{i}^{P}$ (w.r.t. the flow relation) is a transition labelled by $\triangle_{i}^{1}$; it follows from the overall structure that this transition is enabled after $-\$$ occurs. The only (complete) behaviour of $C_{i}^{P}$ (after being enabled) is the sequence of labels shown in Figure 6. And we reason similarly as above.

$$
\begin{gathered}
+\triangle_{i}^{1}+c_{i}-p_{i}^{k_{1}}-\triangle_{i}^{1}+\triangle_{i}^{2}+p_{i}^{k_{1}}-p_{i}^{k_{2}}-\triangle_{i}^{2} \cdots \\
\cdots+\triangle_{i}^{b}+p_{i}^{k_{b-1}}-p_{i}^{k_{b}}-\triangle_{i}^{b}
\end{gathered}
$$

Figure 6: (Linear) behaviour of $C_{i}^{P}$

If the label $+c_{i}$ from $C_{i}^{P}$ belongs to bal, then bal must also contain $+\triangle_{i}^{1}$, and thus also $-\triangle_{i}^{1}$ etc. So if labels of $C_{i}^{P}$ occur in $b a l$, then bal contains an occurrence of some $-p_{i}^{j}$, where $x_{j}$ is a literal of $c_{i}$, such that the 'balancing' occurrence $+p_{i}^{j}$ does not come from $C_{i}^{N}$, and so it must come from $V_{T}^{j}$. We interpret this as 'choosing' the literal $x_{j}$ of $c_{i}$, i.e., as setting $l\left(c_{i}\right)=x_{j}$.

For illustration, Figure 7 shows the STG $S_{\varphi}$ for a simple formula $\varphi$. In fact, it is a slightly different variant of $S_{\varphi}$, using more concurrency and additional signals $T^{j}, F^{j}$ which stress the correspondence with setting variable $x^{j}$ to true or false. (The shaded region shows a balanced sequence, which will be also discussed later.)

We have thus completed the (obviously polynomial) construction of $S_{\varphi}$, and we can easily check that $S_{\varphi}$ is a consistent 1-bounded acyclic marked graph. We have also provided some intuition why the reduction works, i.e., why

$$
\varphi \text { is satisfiable } \Longleftrightarrow S_{\varphi} \text { admits } M_{0} \stackrel{\sigma}{\longrightarrow} M_{1} \stackrel{\tau}{\longrightarrow} M_{2} \text { for some nonempty balanced } \tau \text {. }
$$

Now we summarize the correctness arguments, first informally and then in more detail.

If $\varphi$ is satisfiable, then we 'choose' a satisfying truth assignment $\mathcal{A}$ and for each clause $c_{i}$ we 'choose' a literal $l_{i}$ such that $\mathcal{A}$ makes $l_{i}$ true, where 'choose' has the meaning described above. This leads to a balanced sequence $b a l$. On the other hand, if a balanced sequence bal can be found, then the corresponding 'choice' of literals must be consistent (and so $\varphi$ is satisfiable): if both $x_{j}$ and $\bar{x}_{j}$ are 'chosen', then both $+n_{i}^{j}$ and $-p_{k}^{j}$ appear in $b a l$, and both $V_{T}^{j}$ an $V_{F}^{j}$ must contribute to bal, which, as we have seen, is not possible. A more detailed formulation of these arguments follows.

(" $\Longrightarrow$ ") Suppose that $\varphi$ is satisfiable; we shall show that there is an occurrence sequence $M_{0} \stackrel{\sigma}{\longrightarrow} M_{1} \stackrel{\tau}{\longrightarrow} M_{2}$ in $S_{\varphi}$ such that $\tau$ is nonempty and balanced. Let

$$
l:\left\{c_{1}, c_{2}, \ldots, c_{m}\right\} \rightarrow\left\{x_{1}, \overline{x_{1}}, x_{2}, \overline{x_{2}}, \ldots, x_{n}, \overline{x_{n}}\right\}
$$

be a consistent choice of literals (which must exist since $\varphi$ is satisfiable). 


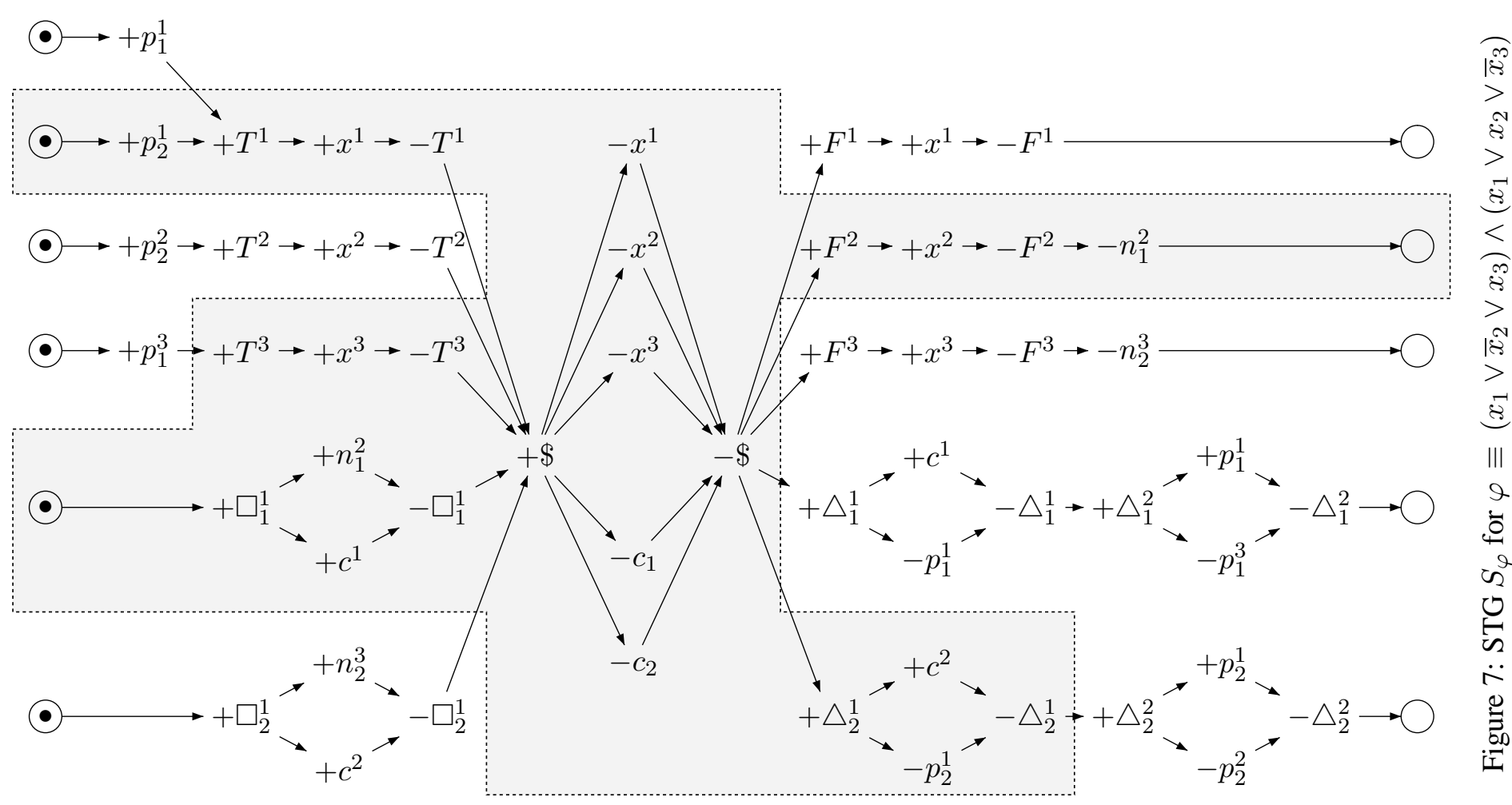


We now define certain sequences $\sigma(X), \tau(X)$ for the appropriate subnets $X$. The required sequence $\sigma$ will then be defined as

$$
\sigma=\sigma\left(V_{T}^{1}\right) \ldots \sigma\left(V_{T}^{n}\right) \sigma\left(C_{1}^{N}\right) \ldots \sigma\left(C_{n}^{N}\right)
$$

and $\tau$ will be defined as $\tau=\tau_{1} \tau_{2} \tau_{3}$, where

$$
\begin{gathered}
\tau_{1}=\tau\left(V_{T}^{1}\right) \ldots \tau\left(V_{T}^{n}\right) \tau\left(C_{1}^{N}\right) \ldots \tau\left(C_{n}^{N}\right) \\
\tau_{2}=\tau\left(N_{\$}\right) \\
\tau_{3}=\tau\left(V_{F}^{1}\right) \ldots \tau\left(V_{F}^{n}\right) \tau\left(C_{1}^{P}\right) \ldots \tau\left(C_{n}^{P}\right)
\end{gathered}
$$

As expected, we define

$$
\tau\left(N_{\$}\right)=+\$-x^{1} \ldots-x^{n}-c_{1} \ldots-c_{m}-\$
$$

For each $j, 1 \leq j \leq n$, we proceed as follows. If $x_{j}$ is in the range of $l$ ( $x_{j}$ was chosen by at least one clause), we define $\tau\left(V_{T}^{j}\right)$ to be a sequence finishing by $+x^{j}$ and otherwise consisting of precisely those $+p_{i}^{j}$ for which $x_{j}=l\left(c_{i}\right) ; \sigma\left(V_{T}^{j}\right)$ contains all others $+p_{i}^{j}$ appearing in $V_{T}^{j}$. And we define $\tau\left(V_{F}^{j}\right)$ as the empty sequence.

If $\overline{x_{j}}$ is in the range of $l$, we define $\sigma\left(V_{T}^{j}\right)$ to be a sequence containing all transitions (i.e, labels) of $V_{T}^{j}$, and we define $\tau\left(V_{T}^{j}\right)$ as empty. But now $\tau\left(V_{F}^{j}\right)$ is nonempty; it starts with $+x_{j}$ and otherwise contains precisely those $-n_{i}^{j}$ for which $\overline{x_{j}}=l\left(c_{i}\right)$.

The above definition is sound since both $x_{j}, \overline{x_{j}}$ can not belong to the range of $l$ ( $l$ is consistent). If none of $x_{j}, \overline{x_{j}}$ belongs to the range of $l$, we can define $\sigma\left(V_{T}^{j}\right)$ as a sequence consisting of all $+p_{i}^{j}$ in $V_{T}^{j}$, and we put $\tau\left(V_{T}^{j}\right)=+x_{j}$ and $\tau\left(V_{F}^{j}\right)$ empty.

Now for each $i, 1 \leq j \leq m$, we proceed as follows. If $l\left(c_{i}\right)=x_{j}$ (thus $\tau\left(V_{T}^{j}\right)$ is nonempty), we define $\tau\left(C_{i}^{P}\right)$ as the sequence

$$
+\triangle_{i}^{1}+c_{i} \ldots-p_{i}^{k_{d}}-\triangle_{i}^{d}
$$

where $k_{d}=j$ (recall Figure 6); thus $+c_{i}$ and $-p_{i}^{j}$ is left to be compensated in $\tau\left(C_{i}^{P}\right)$. And we define $\sigma\left(C_{i}^{N}\right)$ as the whole behaviour of $C_{i}^{N}$, and $\tau\left(C_{i}^{N}\right)$ as empty.

If $l\left(c_{i}\right)=\overline{x_{j}}$ (thus $\tau\left(V_{F}^{j}\right)$ is nonempty), we define

$$
\begin{gathered}
\sigma\left(C_{i}^{N}\right)=+\square_{i}^{1}+n_{i}^{j_{1}} \ldots-n_{i}^{j_{d}}-\square_{i}^{d-1} \\
\tau\left(C_{i}^{N}\right)=+\square_{i}^{d}+n_{i}^{j_{d}} \ldots+c_{i}-\square_{i}^{a}
\end{gathered}
$$

where $j_{d}=j$ (recall Figure 5); thus $+c_{i}$ and $+n_{i}^{j}$ is left to be compensated in $\tau\left(C_{i}^{P}\right)$. And we define $\tau\left(C_{i}^{P}\right)$ as empty.

It is now straightforward to check that the defined sequence $\sigma \tau$ can really occur from $M_{0}$, and that $\tau$ is nonempty and balanced. (For each $j, 1 \leq i \leq m$, either $\tau\left(V_{T}^{j}\right)$ or $\tau\left(V_{F}^{j}\right)$ is nonempty; thus the occurrence $-x^{j}$ of $\tau\left(N_{\$}\right)$ is compensated. For each $i, 1 \leq i \leq m$, either $\tau\left(C_{i}^{N}\right)$ or $\tau\left(C_{i}^{P}\right)$ is nonempty. Suppose that it is $\tau\left(C_{i}^{N}\right)$, the other case being similar. Then $\tau\left(C_{i}^{N}\right)$ is balanced except of the two 'superfluous' occurrences: $+c_{i}$ and $+n_{i}^{j}$, where $l\left(c_{i}\right)=\overline{x_{j}}$. But $+c_{i}$ is compensated by $-c_{i}$ of $\tau\left(N_{\$}\right)$, and $+n_{i}^{j}$ is compensated by $-n_{i}^{j}$ of $\tau\left(V_{F}^{j}\right)$.) 
Remark. The formula $\varphi$ of Figure 7 is satisfiable. The shaded region corresponds to the balanced sequence for the choice $l\left(c_{1}\right)=\overline{x_{2}}, l\left(c_{2}\right)=x_{1}$ (and setting $x_{3}$ to true).

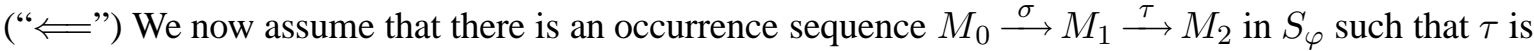
nonempty and balanced; we shall show that $\varphi$ is satisfiable. We can easily check that $\tau$ must contain at least one transition of $N_{\$}$; but this obviously implies that $\tau$ contains all transitions of $N_{\$}$.

Hence $\tau$ can be written

$$
\tau=\tau_{1}^{\prime} \tau_{2}^{\prime} \tau_{3}^{\prime}
$$

where

$$
\tau_{2}^{\prime}=\tau\left(N_{\$}\right)=+\$-x^{1} \ldots-x^{n}-c_{1} \ldots-c_{m}-\$
$$

For each $j, 1 \leq j \leq n$, the occurrence $-x^{j}$ in $\tau_{2}^{\prime}$ must be compensated by $+x^{j}$ in either $\tau_{1}^{\prime}$ or $\tau_{3}^{\prime}$; so precisely one of the nets $V_{T}^{j}$ and $V_{F}^{j}$ contributes to $\tau$. This naturally corresponds to a truth assignment $\mathcal{A}$.

For each $i, 1 \leq j \leq m$, the occurrence $-c_{i}$ in $\tau_{2}^{\prime}$ must be compensated by $+c_{i}$ in either $\tau_{1}^{\prime}$ or $\tau_{3}^{\prime}$. It is thus clear that precisely one of the nets $C_{i}^{N}$ and $C_{i}^{P}$ contributes to $\tau$; in this contribution, precisely one $+n_{i}^{j}$, or $-p_{i}^{j}$ respectively, is left to be compensated. This naturally defines a choice of literals $l$; we will show that this choice $l$ is consistent.

The only possibility how such 'superfluous' $+n_{i}^{j}\left(l\left(c_{i}\right)=\overline{x_{j}}\right)$ is compensated in $\tau$ is by $-n_{i}^{j}$ in $V_{F}^{j}$, which means that $+x_{j}$ of $V_{F}^{j}$ appears in $\tau$ (i.e., $\mathcal{A}\left(x_{j}\right)=0$ ). If it is $-p_{i}^{j}$ which is to be compensated $\left(l\left(c_{i}\right)=x_{j}\right), \tau$ must contain $+p_{i}^{j}$ of $V_{T}^{j}$, which means that $+x_{j}$ of $V_{T}^{j}$ appears in $\tau$ (i.e., $\left.\mathcal{A}\left(x_{j}\right)=1\right)$.

This implies that the above mentioned choice of literals $l$ is indeed consistent, which means that $\varphi$ is satisfiable.

The previous lemma is now used to derive the desired co-NP-hardness results.

Proposition 3.4 Both the CSC problem and the USC problem are co-NP-hard for (consistent) STGs whose underlying nets are 1-bounded acyclic marked graphs.

Proof: We use the STG $S_{\varphi}$ constructed in the proof of Lemma 3.1, recalling that it is a consistent 1-bounded acyclic marked graph; let us denote its (unique) consistent binary encoding by $b$.

Assume now that $S_{\varphi}$ does not have the USC property. This means that there are occurrence sequences

$$
M_{0} \stackrel{\sigma_{1}}{\longrightarrow} M_{1}, \quad M_{0} \stackrel{\sigma_{2}}{\longrightarrow} M_{2}
$$

such that

- $M_{1} \neq M_{2}$

(i.e., $\sigma_{1}$ and $\sigma_{2}$ do not contain the same transitions),

- $b\left(M_{1}\right)=b\left(M_{2}\right)$

We explore the following cases $\mathrm{C} 1$ ), C2), C3), covering all possibilities. 
C1) Some of $\sigma_{1}, \sigma_{2}$ contains $+\$$ but not $-\$$ (turns out impossible) :

In this case, both $\sigma_{1}, \sigma_{2}$ must contain $+\$$ and not $-\$$ (since $b\left(M_{1}\right)=b\left(M_{2}\right)$ ). (I.e., both $M_{1}$, $M_{2}$ are in the 'middle segment'.)

But this is impossible, since we obviously cannot have both $M_{1} \neq M_{2}$ and $b\left(M_{1}\right)=b\left(M_{2}\right)$.

C2) Both $\sigma_{1}, \sigma_{2}$ do not contain $+\$$ or both contain $-\$$ (turns out impossible) :

(I.e., either both $M_{1}, M_{2}$ are in the 'left segment' or both are in the 'right segment'.)

Let us assume the subcase where both $\sigma_{1}, \sigma_{2}$ do not contain $+\$$.

We observe that $\sigma_{1}$ and $\sigma_{2}$ must contain the same transitions of $V_{T}^{j}$, for each $j$ (since $b\left(M_{1}\right)=$ $\left.b\left(M_{2}\right)\right)$.

So there must be a transition of $C_{i}^{N}$ (for some $i$ ), which is, say, in $\sigma_{2}$ but not in $\sigma_{1}$; i.e., $\sigma_{2}$ contains a longer prefix of the behaviour of $C_{i}^{N}$ (cf. Figure 5) than $\sigma_{1}$. But then the difference between the two prefixes must obviously be balanced, which can be easily checked to be impossible.

The subcase where both $\sigma_{1}, \sigma_{2}$ contain $-\$$ can be similarly shown to be impossible.

C3) One of $\sigma_{1}, \sigma_{2}$ does not contain $+\$$, and the other contains $-\$$ :

We can assume that $\sigma_{1}$ does not contain $+\$$ and $\sigma_{2}$ contains $-\$$. (I.e., $M_{1}$ is in the 'left segment', $M_{2}$ is in the 'right segment'.)

This implies that there is (nonempty) $\tau$ such that $M_{0} \stackrel{\sigma_{1}}{\longrightarrow} M_{1} \stackrel{\tau}{\longrightarrow} M_{2}$; necessarily, $\tau$ is balanced. And from the proof of Lemma 3.1 we know that this is possible if and only if $\varphi$ is satisfiable.

Thus cases $\mathrm{C} 1$ ), C2) turn out to be impossible, and C3) is possible if and only if there is a (nonempty) $\tau$ such that $M_{0} \rightarrow^{*} M_{1} \stackrel{\tau}{\longrightarrow} M_{2}$; necessarily, $\tau$ is balanced. Moreover, such $M_{1}, M_{2}$ (with $b\left(M_{1}\right)=$ $b\left(M_{2}\right)$ ) enable different sets of signals, so the CSC property is violated - when viewing all signals as output signals. Therefore recalling Lemma 3.1 finishes the proof.

Proposition 3.5 Both the CSC problem and the USC problem are co-NP hard for live 1-bounded marked graph STGs.

Proof: Consider the USC problem. We reuse the Petri net $S_{\varphi}$ from the proof of Lemma 3.1. We note that the behaviour obtained by firing all transitions of $S_{\varphi}$ is not balanced; i.e., $b\left(M_{0}\right)$ and $b\left(M_{f}\right)$, where $b$ is the consistent boolean encoding and $M_{f}$ is the final marking, differ on some signals.

Remark. For concreteness, these unbalanced signals are $x^{j}, c_{i}, n_{i}^{j_{d}}$ (for $d=2,3, \ldots, a$ ), $p_{i}^{k_{d}}($ for $d=1,2, \ldots, b-1)$.

We define a new STG $S_{\varphi}^{\prime}$ by adding a 'final segment' to $S_{\varphi}$ : we add a fresh signal $f$ and construct a 'linear' net $N_{f}$ with the behaviour

$$
+f \ell_{1} \ell_{2} \cdots \ell_{k}-f
$$


where $\ell_{i}$ are the labels compensating the unbalance of $S_{\varphi}$; they include $-x^{j},-c_{i},+n_{i}^{j_{2}}$, etc.; we note that each nonempty sequence of transitions of $N_{f}$ is unbalanced. The net $N_{f}$ will be prompted in $S_{\varphi}^{\prime}$ after all transitions of $S_{\varphi}$ occur; the final transition of $N_{f}$ will then restore the initial marking $M_{0}$.

Hence $S_{\varphi}^{\prime}$ is an STG whose underlying net is a live and 1-bounded marked graph. It is easy to see that any sequence containing precisely one occurrence of each transition of $S_{\varphi}^{\prime}$ is balanced. Let $b^{\prime}$ be the unique consistent boolean encoding of $S_{\varphi}^{\prime}$.

We show that $S_{\varphi}$ has the USC property iff $S_{\varphi}^{\prime}$ has the USC property, which proves the second part of the proposition.

It is trivial that if $S_{\varphi}$ does not have the USC property, then $S_{\varphi}^{\prime}$ does not have it either. For the other direction, assume that $S_{\varphi}^{\prime}$ does not have the USC property. Then there is a witness of the USCviolation, i.e. two occurrence sequences

$$
M_{0} \stackrel{\sigma_{1}}{\longrightarrow} M_{1}, \quad M_{0} \stackrel{\sigma_{2}}{\longrightarrow} M_{2}
$$

as in the proof of Proposition 3.4.

Let us assume that the witness is minimal in the sense that neither $\sigma_{1}$ nor $\sigma_{2}$ can be shortened. We prove that this minimal witness also corresponds to a USC-violation in the Petri net $S_{\varphi}$. It suffices to show that neither $\sigma_{1}$ nor $\sigma_{2}$ contain a transition labelled by the signal $f$.

Assume that one of $\sigma_{1}$ and $\sigma_{2}$, say $\sigma_{2}$, contains an occurrence of the signal $f$. Since $b^{\prime}\left(M_{1}\right)=$ $b^{\prime}\left(M_{2}\right)$, we can easily check that the assumption $b^{\prime}\left(M_{2}\right)(f)=1$ would force $M_{1}=M_{2}$, a contradiction. So $b^{\prime}\left(M_{2}\right)(f)=0$, which means that the last occurrence of $f$ in $\sigma_{2}$ is $-f$. But then $\sigma_{2}$ can be (rearranged and) written as $\sigma_{2}=\sigma_{2}^{\prime} \sigma$ where $\sigma$ contains precisely one occurrence of each transition of $S_{\varphi}^{\prime}$. This implies $M_{0} \stackrel{\sigma_{2}^{\prime}}{\longrightarrow} M_{2}$, which contradicts our minimality assumption.

Consider now the CSC property. Assume that all signals are output signals. We show that $S_{\varphi}$ has the CSC property iff $S_{\varphi}^{\prime}$ has the CSC property. As in the USC case, it is trivial that if $S_{\varphi}$ does not have the CSC property, then $S_{\varphi}^{\prime}$ does not have it either. For the other direction, assume $S_{\varphi}$ has the CSC property. We have shown in Lemma 3.1 that in this case $S_{\varphi}$ has the USC property as well. So, by the first part of this proof concerning the USC property, $S_{\varphi}^{\prime}$ has the USC property. Since USC implies CSC, $S_{\varphi}^{\prime}$ has the CSC property, and we are done.

We now show the upper bound, a lemma which was already (implicitly) proved in [1].

Lemma 3.2 Both the CSC problem and the USC problem are in co-NP for (bounded or unbounded) marked graph STGs.

Proof: Let $S=\left(N, M_{0}, \ell\right)$ be a normalized and consistent marked graph STG. (We recall that consistency of $S$ can be checked in polynomial time.) It is sufficient to deal with the CSC problem; the claim for the USC problem will follow easily.

We observe that $S$ does not have the CSC property if and only if there are sequences $u_{1}, u_{2}$ such that

- $M_{0} \stackrel{u_{1}}{\longrightarrow} M_{1}, M_{0} \stackrel{u_{2}}{\longrightarrow} M_{2}$,

- $M_{1} \neq M_{2}$,

- for each signal $a$ :

$$
P\left(u_{1}\right)\left(a^{+}\right)-P\left(u_{1}\right)\left(a^{-}\right)=P\left(u_{2}\right)\left(a^{+}\right)-P\left(u_{2}\right)\left(a^{-}\right)
$$


- $M_{1}, M_{2}$ enable different output signals

To check that there is such a 'CSC-violation', a nondeterministic (polynomial) algorithm guesses a place $p$ such that $M_{1}(p) \neq M_{2}(p)$, and guesses further whether $M_{1}(p)>M_{2}(p)$ or $M_{2}(p)>M_{1}(p)$ holds. The algorithm proceeds to guess an output signal $a$, and which of $M_{1}, M_{2}$ enables $a$. Assume w.l.o.g. the guess is that $M_{1}$ enables $a$ and $M_{2}$ does not. The algorithm guesses which places of $M_{1}$ carry at least one token (including all the input places of some transition labeled by $a$ ) and which places of $M_{2}$ carry no token (including at least one input place of each transition labeled by $a$ ). The algorithm translates all these guesses into a system of linear inequalities, guesses an integer solution of polynomial size, and checks in polynomial time that it is indeed a solution. (Variables for transition sequences are replaced by variables for their Parikh vectors, and Claim 3.2 is used.)

Putting together Propositions 3.4 and 3.5 and Lemma 3.2 we obtain:

Theorem 3.2 The CSC problem and the USC problem are co-NP-complete for marked graph STGs, and stay co-NP-hard for live and 1-bounded marked graph STGs as well as for 1-bounded acyclic marked graph STGs.

Remark. Notice that in the marked graphs produced by the reduction from the proof of Lemma 3.1 there are different transitions carrying the same label. The case with injective labelling (each transition has its unique label) might well admit a polynomial algorithm but we leave this problem open here.

\section{Live and bounded free-choice nets}

As already mentioned, [7] shows that consistency can be decided in polynomial time for live and bounded free-choice STGs that are moreover cyclic, meaning that the initial marking is reachable from every reachable marking. It is not known whether the polynomiality result still holds if the cyclicity condition is removed, and we leave this problem open.

We now show co-NP-completeness of the CSC problem and of the USC problem for live and bounded free-choice STGs. Since live and bounded marked graphs are cyclic, Theorem 3.2 gives co-NP-hardness even for cyclic live and bounded free-choice STGs. So we just need to show that the complementary problem is in NP. We proceed similarly as in the marked graph case, first recalling a known result analogous to Claim 3.2; for this we use the following notation:

For a net $N=(P, T, F)$ and $X: T \rightarrow \mathbb{N}$, we denote by $N_{X}=\left(P_{X}, T_{X}, F_{X}\right)$ the subnet of $N$ defined as follows: $T_{X}$ is the set of transitions of $T$ for which $X(t) \geq 1, P_{X}={ }^{\bullet} T_{X} \cup T_{X}^{\bullet}$, and $F_{X}$ is the projection of $F$ on $\left(P_{X} \times T_{X}\right) \cup\left(T_{X} \times P_{X}\right)$. We also recall that $Q \subseteq P$ is a trap in $N=(P, T, F)$ if $Q^{\bullet} \subseteq \bullet$. (If a trap is marked, i.e., has at least one token, it cannot be unmarked). Here we consider only nonempty traps $Q \neq \emptyset$.

Lemma 4.1 ([15]) Let $\left(N, M_{0}\right)$ be a live and bounded free-choice Petri net, and let $C_{N}$ be its incidence matrix. if

An integer vector $X_{0} \geq 0$ is the Parikh vector of a transition sequence enabled at $M_{0}$ if and only

1. $M_{0}+C_{N} \cdot X_{0} \geq 0$, and

2. $M=M_{0}+C_{N} \cdot X_{0}$ marks all traps of $N_{X_{0}}$. 
Theorem 4.1 The CSC problem and the USC problem are co-NP-complete for live and bounded freechoice STGs.

Proof: As mentioned above, co-NP-hardness follows from Theorem 3.2 (even when the Petri nets are also cyclic).

A nondeterministic polynomial algorithm for showing that a given (consistent) live and bounded free-choice STG does not have the CSC property (or the USC property) can be constructed as in the proof of Lemma 3.2, using Lemma 4.1 instead of Claim 3.2.

A little difficulty is the fact that a (nonnegative integer) solution of $M_{0}+C_{N} \cdot X \geq 0$ may not be the Parikh vector of an occurrence sequence. The algorithm handles this problem by guessing (and requiring in the system of inequalities) which components of $X$ are positive and which are zero; then it guesses a subset $P^{\prime}$ of places of $N_{X}$, verifies that $P^{\prime}$ does not contain a trap in $N_{X}$ (which can be easily done in polynomial time) and requires (in the constructed system of inequalities) that $M_{0}+C_{N} \cdot X$ is positive for all places of $N_{X}$ outside $P^{\prime}$.

In the next section we show the importance of the assumption of liveness.

\section{More general nets}

Here we study the complexity of the consistency, CSC, and USC problems for more general classes of STGs.

By a straightforward use of standard techniques of Petri net theory (using the reachability problem for $k$-bounded nets) we can show:

Proposition 5.1 The consistency problem, the CSC problem and the USC problem are PSPACEcomplete for $k$-bounded nets (for any fixed $k$ ).

The relevant proofs can be found in the appendix.

It is worth to note that free-choice does not make this simpler:

Proposition 5.2 The consistency problem for 1-bounded free-choice STGs (not necessarily live) is PSPACE-complete.

Proof: An arbitrary 1-bounded STG can be transformed into a 1-bounded free-choice STG by means of the operation illustrated in Figure $8{ }^{1}$ while preserving consistency.

In more detail: For changing an arbitrary 1-bounded STG into a 1-bounded free-choice STG, we use transformations of the following type:

- into an original arc from a place $p$ to a transition $t$ insert a fresh label $f^{+}$(i.e., add a new freshly labelled transition and an additional place), and

- split $t$ into $t_{1}, t_{2}$, adding a new place $p^{\prime}$ and arcs $t_{1} \rightarrow p^{\prime}, p^{\prime} \rightarrow t_{2}$, where $t_{1}\left(t_{2}\right)$ inherits the current input (output) places of $t$; put $\ell\left(t_{1}\right)=\ell(t)$ and $\ell\left(t_{2}\right)=f^{-}$

\footnotetext{
${ }^{1}$ This operation is closely related to the "releasing arcs"-technique, see e.g. [4]
} 


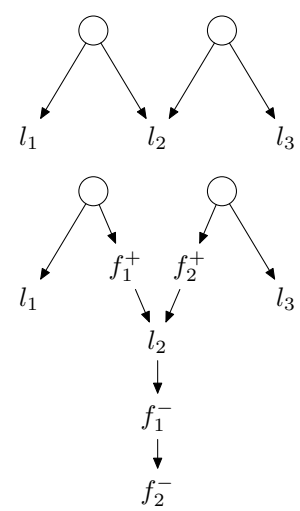

Figure 8: Transforming a 1-bounded STG into a 1-bounded free-choice STG

Figure 8 shows the result of two such transformations, where $f_{2}$ was inserted before $f_{1}$ (in fact, the order of the $f_{i}^{-}$labels is irrelevant); $f_{2}^{-}$now has the original output places of $l_{2}$.

Using sufficiently many such transformations, we can obviously transform each 1-bounded STG $S=\left(N, M_{0}, \ell\right)$ into $\bar{S}=\left(\bar{N}, \overline{M_{0}}, \bar{\ell}\right)$ which is 1-bounded and free-choice; $\overline{M_{0}}$ coincides with $M_{0}$ on places from $N$ and is 0 elsewhere.

Important is that $S$ is consistent iff $\bar{S}$ is consistent. For this, it is sufficient to show that using the described transformation, i.e. the step performed for one arc and changing an intermediate $S^{\prime}$ to $S^{\prime \prime}$, keeps the (in)consistency untouched. We can easily note that if $S^{\prime}$ is inconsistent then so is $S^{\prime \prime}$. And 1-boundedness guarantees that $S^{\prime \prime}$ can not be inconsistent due to the added $f^{+}, f^{-}$; thus an inconsistency witness in $S^{\prime \prime}$ naturally 'translates' into $S^{\prime}$.

Using reductions from and to the reachability problem of general Petri nets, we can show

Proposition 5.3 The consistency problem and the CSC problem for general STGs are decidable but EXPSPACE-hard.

The proofs are also in the appendix.

\section{Conclusions and related work}

We have explored the complexity of the consistency and the CSC problem for several classes of STGs. The main result shows that deciding the CSC property is co-NP-complete even for 1-bounded and acyclic marked graph STG and for 1-bounded and live marked graph STGs. The same result holds for the USC property. This result explains why none of the existing approaches for checking the USC or the CSC property in marked graph STGs is polynomial or complete.

In [14] the USC property was studied for live and 1-bounded marked graph STGs with injective labeling (i.e., one up-transition and one down-transition per signal). A sufficient condition for the USC property to hold is presented, and it is shown that it can be checked in polynomial time. The condition is conjectured to be also necessary, which would imply that checking the USC property is polynomial. The reduction used in our NP-completeness result transforms a formula into an STG in which several signals have two up- and two down-transitions, and so it does not apply to this case. The complexity of the USC property for this particular case is left for future research. 
In [16] the result of [14] is extended to the case in which the STG may have several up- and down-transitions per signal. The paper presents a generalization of the sufficient condition of [14]. Our NP-completeness result shows that if $\mathrm{P} \neq \mathrm{NP}$ then the condition is not necessary or it cannot be checked in polynomial time, or both. In fact, we conjecture that the condition is neither necessary, nor can be checked in polynomial time (it requires to establish a property for a potentially exponential number of objects).

In [17] it is shown that a live and 1-bounded marked-graph STGs violates the USC property iff the STG has a so-called complementary path. The paper proposes an algorithm that searches for such paths. The worst-case complexity of the algorithm is exponential, and by our result this is unavoidable unless $\mathrm{P} \neq \mathrm{NP}$.

In [11] a polynomial algorithm is presented that detects all violations of the CSC property in a live and bounded free-choice STG. However, the algorithm may also give false positives, i.e., it may detect false violations. Our result shows that if $\mathrm{P} \neq \mathrm{NP}$ then every polynomial algorithm must produce false positives or false negatives.

In [1] a procedure is described that, given a marked-graph STG, constructs in polynomial time an Integer Linear Programming (ILP) problem such that the STG violates the CSC property if and only if the problem has a solution. Our result shows that, unless $\mathrm{P} \neq \mathrm{NP}$, ILP is necessary, and cannot be replaced by ordinary Linear Programming (recall that Linear Programming problems can be solved in polynomial time).

In $[9,10]$ it is shown how to check the CSC property for arbitrary bounded STGs using net unfoldings and ILP-solvers or SAT-solvers. Given a bounded STG $S$, an object is constructed called the unfolding of $S$. This unfolding is used to generate an ILP problem (a boolean formula) such that $S$ violates the CSC property iff the ILP problem has a solution (iff the formula is satisfiable). If $S$ is a live and 1-bounded marked graph, then the unfolding of $S$ has polynomial size in $S$ ([5], Theorem 4.14). This shows that, even for marked graphs, ILP-solvers or SAT-solvers are unlikely to be replaceable by other tools with polynomial running time: if $\mathrm{P} \neq \mathrm{NP}$, then no polynomial algorithm taking the unfolding of $S$ as input can decide the CSC or the USC property.

Finally, it could be argued that the important problem in practice is not to decide whether a given STG satisfies the CSC property, but to transform an STG that does not satisfy the CSC property into another one that does. In [2] an automatic, very efficient procedure for such a transformation is presented. Unfortunately, the procedure adds many additional signals (one per place of the STG), and so in most cases its output is only useful as a first approximation to the design. The optimization of this first approximation has to be carried out by a (possibly automatic) trial and error procedure in which a candidate for an optimized STG is guessed. The candidate must be checked for the CSC property, which brings us back to the problem discussed in this paper.

Acknowledgments. The first author thanks Jordi Cortadella and José Carmona for helpful discussions.

\section{References}

[1] J. Carmona and J. Cortadella. ILP models for the synthesis of asynchronous control circuits. In 2003 International Conference on Computer-Aided Design (ICCAD'03), November 9-13, 2003, San Jose, CA, USA, pages 818-826. IEEE Computer Society / ACM, 2003.

[2] J. Carmona, J. Cortadella, and E. Pastor. A structural encoding technique for the synthesis of asynchronous circuits. In Proc. Int. Conf. on Application of Concurrency Theory to System Design, pages 157-166. IEEE Computer Society, 2001. 
[3] T.-A. Chu. Synthesis of Self-Timed VLSI Circuits from Graph-theoretic Specifi cations. PhD thesis, MIT, 1987.

[4] J. Desel and J. Esparza. Free Choice Petri Nets, volume 40 of Cambridge Tracts in Theoretical Computer Science. Cambridge University Press, 1995.

[5] J. Esparza. Model checking using net unfoldings. Science of Computer Programming, 23:151-195, 1994.

[6] J. Esparza. Decidability and complexity of Petri net problems - an introduction. In G. Rozenberg and W. Reisig, editors, Lectures on Petri Nets I: Basic Models. Advances in Petri Nets, number 1491 in Lecture Notes in Computer Science, pages 374-428, 1998.

[7] J. Esparza. A polynomial-time algorithm for checking consistency of free-choice signal transition graphs. Fundamenta Informaticae, 62(2):197-220, 2004.

[8] J. Esparza, P. Jančar, and A. Miller. On the complexity of consistency and complete state coding for signal transition graphs. In Proceedings of the 6th International Conference on Application of Concurrency to System Design (ACSD 2006), Turku, Finland, June 2006. IEEE Computer Society.

[9] V. Khomenko, M. Koutny, and A. Yakovlev. Detecting State Coding Conficts in STGs Using Integer Programming. In Proc. of the Design, Automation and Test in Europe Conference and Exhibition, pages 338-345. IEEE Computer Society, 2002.

[10] V. Khomenko, M. Koutny, and A. Yakovlev. Detecting State Coding Conficts in STG Unfoldings using SAT. In Proc. of the 4th Int. Conf. on Application of Concurrency to System Design, pages 16-25. IEEE Computer Society, 2004.

[11] E. Pastor and J. Cortadella. Polynomial algorithms for the synthesis for hazard-free circuits from signal transition graphs. In 1993 International Conference on Computer-Aided Design (ICCAD'93), Santa Clara, CA, USA, pages 250-254. IEEE Computer Society / ACM, 1993.

[12] L. Rosenblum and A. Yakovlev. Signal graphs: from self-timed to timed ones. In Proc. Int. Workshop on Timed Petri nets, pages 199-207. IEEE Computer Society, 1985.

[13] A. Schrijver. Theory of Linear and Integer Programming. Wiley, 1986.

[14] P. Vanbekbergen, F. Catthoor, G. Goossens, and H. D. Man. Optimized synthesis of asynchronous control circuits from graph-theoretic specifications. In 1990 International Conference on Computer-Aided Design (ICCAD'90), pages 184-197. IEEE Computer Society, 1990.

[15] H. Yamasaki, J. Huang, and T. Murata. Reachability analysis of petri nets via structural and behavioral classifications of transitions. Petri Net Newsletter, (60):5-21, 2001.

[16] C. Ykman-Couvreur, B. Lin, G. Goossens, and H. D. Man. Synthesis and optimization of asynchronous controllers based on extended lock graph theory. In 4th European Conference on Design Automation, Paris, France, pages 512-517. IEEE Computer Society, 1993.

[17] M. Yu and P. Subrahmanyam. A new approach for checking the unique state coding property of signal transition graphs. In Proc. 3rd Int. European Conference on Design Automation, pages 312-321. IEEE Computer Society, 1992.

\section{Appendix (Bounded nets and general nets)}

This appendix provides proofs for the results mentioned in Section 5. I.e., it demonstrates the hardness of the consistency problem and the CSC problem in the general case, where the underlying net of an STG can be an arbitrary Petri net, and in the subcase when a bound on the number of tokens in each place is given (which can be viewed as 'capacity'). This is done by showing suitable polynomial reductions from the reachability problem to the consistency problem and to the CSC problem respectively.

In fact, the reachability problem can also provide an upper bound, so the consistency and CSC problems can be roughly viewed as equivalent with the reachability (w.r.t. the computational complexity). We also clarify the case of consistency in more detail, by showing a relation to the fireability problem (which is straightforwardly equivalent to the coverability problem). 
The proof ideas use the usual techniques, so we do not describe them very formally nor in great detail. For completeness, we start by recalling definitions and the known complexity results for reachability and fireability. (Precise references can be found, e.g., in [6].)

The reachability problem $(\mathrm{RP})$

Instance: a Petri net $\left(N, M_{0}\right)$ and a marking $M$.

Question: Is $M_{0} \longrightarrow^{*} M$ ?

Theorem 7.1 For (general) Petri nets, RP is decidable and EXPSPACE-hard. For k-bounded nets (for any fixed $k$ ), RP is PSPACE-complete.

The fireability problem (FP)

Instance: a Petri net $\left(N, M_{0}\right)$ and a transition $t$.

Question: Is $t$ fireable (i.e., is there some $M$ such that $M_{0} \longrightarrow^{*} M \stackrel{t}{\longrightarrow}$ ) ?

Theorem 7.2 For (general) Petri nets, FP is EXPSPACE-complete. For $k$-bounded nets (for any fixed $k$ ), FP is PSPACE-complete.

\subsection{Nonreachability reduces to consistency}

We show how an instance $\left(N, M_{0}\right), M_{1}$ of the reachability problem in general nets can be transformed into an STG which is consistent if and only if $M_{1}$ is not reachable in $\left(N, M_{0}\right)$. Moreover, if $\left(N, M_{0}\right)$ is 1-bounded then the constructed STG is also 1-bounded. In addition, we clarify the difference between conditions (1) and (2) of Proposition 2.1 on one hand and condition (3) on the other hand. Conditions (1), (2) turn out to be equivalent to the fireability problem; it is condition (3) which is as difficult as reachability.

We start with a simple construction that we use several times:

Construction 1: Given a net $\left(N, M_{0}\right)$, we denote by $S_{\left(N, M_{0}\right)}$ the STG obtained as follows:

- As the set of signals of $S_{\left(N, M_{0}\right)}$ we take the set of transitions of $N$.

- In $N$, we replace every transition $t$ by a place $p_{t}$ and two transitions $t_{1}, t_{2}$, labeled by $t^{+}, t^{-}$, respectively. Transition $t_{1}$ inherits the input places of $t$, and has $p_{t}$ as the unique output place; transition $t_{2}$ has $p_{t}$ as the unique input place and inherits the output places of $t$.

- We add a (run-)place $r$, and an $\operatorname{arc} r \rightarrow t_{1}$ and $t_{2} \rightarrow r$, for every transition $t$ of $N$.

- The initial marking of $S_{\left(N, M_{0}\right)}$ coincides with $M_{0}$ on the places inherited from $N$; moreover, $r$ carries 1 token, and places $p_{t}$ are empty.

Observation 7.1 The $S T G S_{\left(N, M_{0}\right)}$ is consistent, and it tightly simulates the behaviour of $\left(N, M_{0}\right)$.

Proposition 7.1 The reachability problem for general Petri nets (for $k$-bounded Petri nets) is polynomially reducible to the inconsistency problem for general STGs (for $k$-bounded STGs).

Proof: Assume an instance of the reachability problem: $\left(N, M_{0}\right), M_{1}$. We consider the following construction of an STG $S$ : 
- Start with $S_{\left(N, M_{0}\right)}$ as described in Construction 1.

- Add a transition $t_{f}$ labelled by a fresh label $f^{+}$, and an arc from (the run-place) $r$ to $t_{f}$. (Thus the added $t_{f}$ can fire at most once, by which a dead marking is reached, corresponding to a reachable marking of $N$.)

- Add a new (starting) place $s$; the initial marking of $S$ will put 1 token in $s$; all other places (including $r$ ) will be initially empty.

- Add a transition $z_{1}$, labelled by a fresh $a^{+}$. It takes the token from $s$ and installs $M_{1}$ in the places inherited from $N$. (Marking $M_{1}$ thus becomes 'frozen'.)

- Finally add transitions $z_{2}, z_{3}$, labelled with $a^{+}$and $a^{-}$, a place $p_{z}$, and the arcs

$$
s \rightarrow z_{2}\left(a^{+}\right) \rightarrow p_{z} \rightarrow z_{3}\left(a^{-}\right) \rightarrow r
$$

as well as additional arcs from $z_{3}$ which install $M_{0}$ in the places inherited from $N$.

We observe that the constructed $S$ can start either with firing $z_{1}$ (label $a^{+}$), reaching the 'frozen' $M_{1}$, or with firing $z_{2} z_{3}\left(a^{+} a^{-}\right)$after which it behaves like $S_{\left(N, M_{0}\right)}$, with a possibility to 'freeze' any reachable marking of $\left(N, M_{0}\right)$. We also note that if $\left(N, M_{0}\right)$ is $k$-bounded then $S$ is also $k$-bounded.

It is clear that $S$ can not provide any inconsistency witness $(M, a)$ of the form (1) and (2) of Proposition 2.1; there might be a witness satisfying (3) but this happens if and only if $M_{1}$ is reachable in $\left(N, M_{0}\right)$.

The previous reduction was based on condition (3). For completeness, we show that the existence of inconsistency witness of form (1) or (2) is 'easier', namely polynomially equivalent to the fireability problem.

Claim 7.1 The problem of deciding, given an STG $S$, if there is a pair $(M, a)$ satisfying conditions (1) or (2) of Proposition 2.1 is polynomially equivalent to the fireability problem.

Proof: We first show that the problem if $S$ provides a pair $(M, a)$ satisfying (1) can be reduced to the fireability problem.

Let us fix $a$ (a signal), and define

- $S_{a}^{+}$is a 'copy' of $S$ from which we remove all transitions labelled by $a^{-}$together with their adjacent arcs, and we add a ('run'-)place $r_{a}^{+}$with 1 token. For every transition $t$ of $S_{a}^{+}$which is not labeled by $a^{+}$we add $\operatorname{arcs} r_{a}^{+} \rightarrow t, t \rightarrow r_{a}^{+}$; in the case of $t$ labelled $a^{+}$we only add $r_{a}^{+} \rightarrow t$.

We observe that $S_{a}^{+}$behaves like $S$ until a first occurrence of an $a$-label; this $a$-label must be $a^{+}$, and the computation of $S_{a}^{+}$is thus finished.

Similarly we proceed for $a^{-}$:

- $S_{a}^{-}$is a 'copy' of $S$ from which we remove all transitions labelled by $a^{+}$together with their adjacent arcs, and we add a ('run'-)place $r_{a}^{-}$with 1 token. For every transition $t$ of $S_{a}^{-}$which is not labeled by $a^{-}$we add $\operatorname{arcs} r_{a}^{-} \rightarrow t, t \rightarrow r_{a}^{-}$; in the case of $t$ labelled $a^{-}$we only add $r_{a}^{-} \rightarrow t$. 
We now put STGs $S_{a}^{+}, S_{a}^{-}$side by side. We add new places $p_{a}^{+}, p_{a}^{-}$, initially empty, and a new transition $t_{f}^{a}$ with arcs $p_{a}^{+} \rightarrow t_{f}^{a}, p_{a}^{-} \rightarrow t_{f}^{a}$. Moreover, for each $t$ in $S_{a}^{+}$labelled by $a^{+}$we add $t \rightarrow p_{a}^{+}$, and for each $t$ in $S_{a}^{-}$labelled by $a^{-}$we add $t \rightarrow p_{a}^{-}$.

We have thus got a Petri net $\left(N, M_{0}\right)$ where $t_{f}^{a}$ is fireable if and only if the initial marking $M$ of $S$ satisfies (1).

To reach our goal, we still modify the net $\left(N, M_{0}\right)$ :

- Add a new (run-)place $r$, initially with a token, and let $r_{a}^{+}, r_{a}^{-}$be initially empty.

- For each transition $t$ of $S$, add an additional copy of $t$ (to $\left(N, M_{0}\right)$ ), with the $\operatorname{arcs} r \rightarrow t, t \rightarrow r$. For each arc $p \rightarrow t$ in $S$, add arcs $p_{1} \rightarrow t, p_{2} \rightarrow t$ where $p_{1}, p_{2}$ are the copies of $p$ in $S_{a}^{+}, S_{a}^{-}$ respectively. Similarly for the ouput arcs $t \rightarrow p$.

- Finally add a transition $t_{a}$, with $\operatorname{arcs} r \rightarrow t_{a}, t_{a} \rightarrow r_{a}^{+}, t_{a} \rightarrow r_{a}^{+}$.

We observe that the arisen net $\left(N^{\prime}, M_{0}^{\prime}\right)$ in the first phase simulates $S$ synchronously on both the places in $S_{a}^{+}$and the places in $S_{a}^{-}$. To enable $t_{f}^{a}$, this first phase must stop by firing $t_{a}$ (which unmarks $r$ and marks $r_{a}^{+}, r_{a}^{-}$). Transition $t_{f}^{a}$ can then indeed be enabled if and only if the corresponding $M$, reachable in $S$ and copied in both $S_{a}^{+}$and $S_{a}^{-}$, satisfies (1).

Thus we have described a polynomial algorithm which, given an STG $S$ and signal $a$, constructs $\left(N^{\prime}, M_{0}^{\prime}\right)$ so that $S$ has an inconsistency witness $(M, a)$ satisfying (1) iff $t_{f}^{a}$ is fireable in $\left(N^{\prime}, M_{0}^{\prime}\right)$. The construction can be completed by subnets $S_{b}^{+}$and $S_{b}^{-}$for all signals $b$, and some straightforward modifications, one of them ensuring that firing any $t_{f}^{b}$ will enable an additional distinguished transition $t_{f}$.

By such a technique, we can extend the overall construction to show that

there is a polynomial algorithm which, given an STG $S$, constructs a net $\left(N_{S}, M_{S}\right)$, with a distinguished transition $t_{f}$, so that $S$ has an inconsistency witness $(M, a)$ satisfying (1) or (2) iff $t_{f}$ is fireable in $\left(N_{S}, M_{S}\right)$.

For the other direction, assume an instance $\left(N, M_{0}\right), t$ of the fireability problem. We start with constructing $S_{\left(N, M_{0}\right)}$ by Construction 1 ; then we add a new transition $z$, whose only input place is $p_{t}$ and whose label is $t^{+}$. it is clear that the so constructed STG $S$ has some $(M, a)$ satisfying (1), or (2), iff $t$ is fireable in $\left(N, M_{0}\right)$.

\subsection{Nonreachability reduces to CSC}

Proposition 7.2 The reachability problem for general Petri nets (for $k$-bounded Petri nets) is polynomially reducible to the negation of the CSC-problem for general STGs (for k-bounded STGs).

Proof: We use the single-place-zero reachability problem. So an instance is $\left(N, M_{0}\right)$ and a place $p_{0}$, and the question is if there is a reachable $M$ with $M\left(p_{0}\right)=0$. Given such an instance, we construct a (consistent) STG $S$ as follows:

- We start with the (consistent) STG $S_{\left(N, M_{0}\right)}$ from Construction 1; recall that it has a run-place $r$. 
- We now add two transitions $t_{1}, t_{2}$, labelled by fresh $a^{+}, a^{-}$, places $p_{1}, r_{1}$, and the depicted arcs:

$$
r \rightarrow t_{1}\left(a^{+}\right) \rightarrow p_{1} \rightarrow t_{2}\left(a^{-}\right) \rightarrow r_{1}
$$

- We still add two further transitions, $t_{3}, t_{4}$ labelled by fresh $b+, b-$, a place $p_{2}$, and the depicted arcs:

$$
r \rightarrow t_{3}(b+) \rightarrow p_{2} \rightarrow t_{4}(b-), t_{4} \rightarrow r_{1}, t_{4} \rightarrow p_{0}
$$

- Finally we add a new transition $t_{5}$ labelled by $o+$, where $o$ is defined as the only output signal; and we add the $\operatorname{arcs} r_{1} \rightarrow t_{5}, p_{0} \rightarrow t_{5}$.

We observe that the constructed $S$ is still consistent, we denote the consistent boolean encoding by $b$.

Now assume that $\left(N, M_{0}\right)$ can reach $M$ such that $M\left(p_{0}\right)=0$. Then in $S=\left(N^{\prime}, M_{0}^{\prime}, \ell\right)$ we have

- $M_{0}^{\prime} \longrightarrow^{*} M^{\prime}$ where $M^{\prime}\left(p_{0}\right)=0$

- $M^{\prime} \stackrel{a^{+} a^{-}}{\longrightarrow} M_{1}$ where $M_{1}\left(p_{0}\right)=0$

- $M^{\prime} \stackrel{b^{+} b^{-}}{\longrightarrow} M_{2}$ where $M_{2}\left(p_{0}\right)=1$

So $M_{1} \neq M_{2}$ and necessarily $b\left(M_{1}\right)=b\left(M_{2}\right)$; but $M_{1}$ does not enable $o^{+}$and $M_{2}$ does. Hence $S$ does not have the CSC-property.

On the other hand, if all reachable $M$ in $\left(N, M_{0}\right)$ satisfy $M(p) \geq 1$ then $S$ obviously has the CSC-property.

Finally we note that if $\left(N, M_{0}\right)$ is $k$-bounded then the STG $S$ is 'almost' $k$-bounded. The only problem (increasing to $k+1$ ) can be caused by the arc $t_{4} \rightarrow p_{0}$. But we can replace it by an arc $t_{4} \rightarrow p^{\prime}$ for a new place $p^{\prime}$, and add a further transition $t_{5}^{\prime}$ labelled by $o^{+}$, with the $\operatorname{arcs} r_{1} \rightarrow t_{5}^{\prime}$, $p^{\prime} \rightarrow t_{5}^{\prime}$.

\subsection{PSPACE-completeness for bounded nets}

Let us consider $k$-bounded STGs. Propositions 7.1 and 7.2 show that both the consistency problem and the CSC problem are PSPACE-hard. For showing that these problems are in PSPACE, it is sufficient to consider nondeterministic algorithms for the complementary problems (recall that PSPACE=NPSPACE). But such algorithms are obvious; so we have

Proposition 7.3 Both the consistency problem and the CSC problem are PSPACE-complete for (explicitly-)bounded nets.

\subsection{Reduction to reachability}

To provide some upper bound on the complexity of the consistency and CSC problems in the general case, we show reductions to the reachability problem. By a reduction we mean showing how an instance (of the consistency or CSC problem) can be answered by solving possibly several instances of the reachability problem, all of them being constructed in polynomial time. 
Proposition 7.4 Consistency problem (for general STGs) is reducible to the reachability problem.

Proof: Due to Claim 7.1, it sufficient to handle condition (3) of Proposition 2.1. Given an STG, to decide if there is $M$ reachable by $w_{1} a^{+} u$ and by $w_{2} a^{-} v$ for some $a$-free sequences $u, v$, we can let run two copies $S^{\prime}, S^{\prime \prime}$ of $S$ independently (each having its own run-place). $S^{\prime}$ has a possibility to 'freeze' a marking reached by a sequence where $a^{+}$was the last $a$-transition, $S^{\prime \prime}$ has a possibility to 'freeze' a marking reached by a sequence where $a^{-}$was the last $a$-transition. In the final phase, the markings in both copies $S^{\prime}, S^{\prime \prime}$ will be 'compared': for each place of $S$, a transition taking from $p^{\prime}, p^{\prime \prime}$, the copies of $p$ in $S^{\prime}, S^{\prime \prime}$, can fire as long as possible. The everywhere-zero marking can thus be reached iff the markings reached in $S^{\prime}$ and $S^{\prime \prime}$ coincide.

Proposition 7.5 The CSC problem (for general STGs) is reducible to the reachability problem.

\section{Proof:}

Let us have an STG $S=\left(N, M_{0}, \ell\right)$ (which can be supposed to be consistent). For each signal $a$, we add places $p_{a+}$ and $p_{a-}$, and we solve which of $a^{+}, a^{-}$can be enabled first. In the first case, we put 1 token in $p_{a-}$ and 0 tokens in $p_{a+}$, and in the second case vice versa. And we add arcs so that each $a^{+}$-transition takes a token from $p_{a-}$ and puts a token in $p_{a+}$, and each $a^{-}$-transition takes from $p_{a+}$ and puts in $p_{a-}$.

So the modified net faithfully simulates the original $S$; moreover, each reachable marking contains explicit information about the current (consistent) binary encoding.

We can again use two copies of $S$, use run-places for distinct phases of computation etc., so that this allows to choose any two reachable markings $M_{1}, M_{2}$ after which it will be guaranteed that a specified (sub)marking will be reachable iff $M_{1}, M_{2}$ have the same binary encoding and one enables a certain output signal but the other not. (We can solve this for each output signal separately.) 